\title{
Therapeutic Effects of CXCL9-overexpressing Human Umbilical Cord Mesenchymal Stem Cells on Liver Fibrosis in Rats
}

\section{Yang Li}

Hebei Medical University

\section{Xueshuai Ye}

Hebei Medical University

\section{Xueqian Zhang}

Hebei General Hospital

\section{Ziqi Cai}

Hebei Engineering Technology Researh Center for Cell Therapy

\section{Li Shen}

Hebei Engineering Technology Research Center for Cell Therapy

\section{Mengya Zhang}

Hebei Engineering Technology Research Center for Cell Therapy

\section{Wentao Zhang}

Hebei Medical University

Jianhui Cai ( $\square$ jianhuicai200l@163.com )

Hebei General Hospital https://orcid.org/0000-0001-8744-3565

\section{Research Article}

Keywords: Umbilical cord mesenchymal stem cells, CXCL9, liver fibrosis, cell therapy, gene therapy

Posted Date: July 28th, 2021

DOI: https://doi.org/10.21203/rs.3.rs-742448/v1

License: (c) (1) This work is licensed under a Creative Commons Attribution 4.0 International License. Read Full License

Version of Record: A version of this preprint was published at Biochemical and Biophysical Research Communications on November 1st, 2021. See the published version at https://doi.org/10.1016/j.bbrc.2021.10.078. 


\section{Abstract}

Background: Umbilical cord mesenchymal stem cells (UC-MSCs) transplantation have become a promising treatment for liver fibrosis. However, UC-MSCs have limited anti-fibrosis ability, and their homing ability of UC-MSCs to the injured liver sites appears to be poor. In this study, we aimed to determining if overexpression of CXCL9 could have the synergistic anti-fibrosis effect with UC-MSCs, and whether it can promote the homing ability of UC-MSCs.

Methods: Overexpression of CXCL9 in UC-MSCs (CXCL9-UC-MSCs) was attained by transfection of naive UC-MSCs with the lenti-CXCL9-mCherry. The impact of transplanted CXCL9-UC-MSCs on both repairing of liver fibrosis and homing was evaluated and compared with lenti-mCherry empty vector transfected UCMSCs (control UC-MSCs).

Results: After puromycin screening, UC-MSCs could stably express CXCL9 without affecting the stem and differentiation ability of UC-MSCs. In addition, biochemical analysis showed that the liver function of CXCL9-UC-MSCs was significantly increased in comparison with that of control UC-MSCs $(P<0.05)$. Futhermore, histopathology after 4 weeks of cell therapy demonstrated that the content of collagen fibers decreased obviously, the pseudo-lobules almost disappeared, and the morphology of hepatic lobules was basically normal. Frozen sections were performed 24 hours and 4 weeks after the cell injection. It can be seen that the fluorescence expression of the CXCL9-UC-MSCs group was significantly higher than that of the control UC-MSCs group, which proved that CXCL9-UC-MSCs have a stronger chemotactic ability, and can stay longer than control UC-MSCs in the injured liver.

Conclusion: Overexpression of CXCL9 improves the efficacy of UC-MSC therapy for liver fibrosis repair, thereby promoting the homing and staying of UC-MSCs to injured hepatic sites in a rat model of liver fibrosis.

\section{Background}

Liver fibrosis is a intersection of the end-stage chronic liver disease caused by various reasons. With the progress of the disease, it will progressively worsen to cirrhosis, decompensation of cirrhosis or even liver cancer, which is a serious threat to human's health and life. Orthotopic liver transplantation (OLT) is an important treatment for end-stage liver disease. However, it is difficult to widely carry out OLT due to few donors, high risk, immune rejection and complications. Therefore, seeking new effective treatment methods has become the focus of end-stage liver disease, such as cell therapy and gene therapy. Umbilical cord mesenchymal stem cells (UC-MSCs) are pluripotent stem cells, which have a wide range of sources and low immunogenicity. In addition to their differentiation activity and self-renewal ability, they also have paracrine effects improving the disease microenvironment [1-4]. Under the induction of specific conditions, these cells can also differentiate into osteoblasts, adipocytes and hepatocytes [5]. Many studies have applied Umbilical cord mesenchymal stem cells can be treatment to liver fibrosis and have shown effectiveness of anti-fibrosis. Unfortunately, there is still uncertainty about the effect. One of 
the reasons is that it is difficult to accurately homing to the target organ after intravenous transplantation, which affects the therapeutic effect of UC-MSCs [6]. Therefore, using gene technology to modify mesenchymal stem cells to further improve their chemotaxis and repair ability may provide a more effective treatment for chronic liver diseases such as liver fibrosis.

Chemokines are a class of chemotactic molecules with cytokine function, which play a role in regulating inflammatory response in many tissues. In acute and chronic liver diseases, chemokines usually indirectly participate in the injury response by regulating the infiltration of immune cells (monocytes, $T$ cells) into the liver. Many studies have shown that chemokines can act directly on hepatocytes and stellate cells, and participate in the response to inflammation and fibrosis damage. In liver fibrosis, a direct inhibitory effect of CXCL9 on collagen secretion of hepatic stellate cells (HSC) was identified, and CXCL9 was also a strong counter-regulatory molecule of VEGF-driven aberrant liver vascularization and perfusionin vitro and in vivo [7]. Interestingly, CXCL9 also have been shown to influence the adhesion, crawling and spreading between MSCs and aortic endothelial cells [8].

In this study, we aim to establish the CXCL9-UC-MSCs by overexpression of CXCL9, and to determine whether CXCL9-UC-MSCs could improve their capability for ameliorating liver fibrosis, thereby promoting homing of UC-MSCs to rat livers and provide experimental basis for UC-MSCs in the treatment of liver fibrosis and other chronic liver diseases in the future.

\section{Methods}

\section{Isolation culture of UC-MSCs}

The UC-MSCs were isolated and cultivated with the tissue explants adherent method in vitro. Human umbilical cord were obtained from Hebei General Hospital with patients' informed consent, and in compliance with national legislation. All experimental protocols were approved by the Ethics Committee of Hebei General Hospital. The cord is washed with cold phosphate-buffered saline (PBS) after removal of umbilical arteries and veins. Then, cut the cord into small pieces $(2-5 \mathrm{~mm})$ and cultured in a $10 \mathrm{~cm}^{2}$ culture dish supplemented with Clin-SFM®-Human MSC (Beijing Clin-Biotechnology, Beijing, China). Cells are incubated at $37^{\circ} \mathrm{C}$ at an atmosphere of $5 \% \mathrm{CO}_{2}$ with saturated humidity. The UC-MSCs from the third passage were selected and then a total of $10^{6} / 100 \mathrm{uL}$ cells were labeled with different antibodies, including CD45, CD34, CD73 and CD90 (BD Biosciences, CA, USA). Subsequently, the solution was stored in the dark for $30 \mathrm{~min}$. After washing with PBS, the cells were subjected to analysis and validation of specific markers by flow cytometry.

\section{Adipogenic and osteogenic differentiation}

UC-MSCs were plated at a density of $1 \times 10^{5}$ cells in 6 well plates and incubated at $37^{\circ} \mathrm{C}$ in a humidified atmosphere of $5 \%$ CO2. At $100 \%$ confluence, we performed adipogenic differentiation by treating UCMSCs with Human Umbilical Cord Mesenchymal Stem Cell Adipogenic Differentiation Kit (Cyagen 
Biosciences, Guangzhou, China) for 3 weeks according to protocol, followed by staining with oil red 0 to detect lipids.

To investigate the osteogenic differentiation capacity of UC-MSCs, cells in 6 well plates were treated with Mesenchymal Stem Cell Osteogenic Differentiation Kit (Cyagen Biosciences, Guangzhou, China) at $60 \%-70 \%$ confluence. The medium was changed every 3-4 days. After 3 to 4 weeks, the cells were stained with Alizarin Red S, rinsed with water and examined by microscopy.

\section{Peraperation of CXCL9-UC-MSCs and control UC-MSCs}

To establish CXCL9-UC-MSCs and control UC-MSCs, the lenti-CXCL9-mCherry or lenti-mCherry empty vector was used to transfect naive UC-MSCs at passage 3 respectively. The lenti-CXCL9-mCherry was purchased from GenePharma (Suzhou, Jiangsu, China ). In preparatory experiment, we transfected UCMSCs with $\mathrm{MOI}=1,10,20,50,80,100$ in 96 well plates,polybrene with concentration of $5 \mathrm{ug} / \mathrm{ml}$ was added to enhance the infection efficiency. After 72-96 hours of infection, the number of infection with the best cell condition and the highest expression of mCherry was selected as the amount of virus used in the formal infection experiment.

We conducted the formal test in 6 well plates. UC-MSCs were plated in a 6 well plates at $1 \times 10^{5}$ cells/well. After 24 hours of transfection $(\mathrm{MOI}=50)$, the cells were cultured in complete medium continuously. 72 hours after infection, CXCL9-UC-MSCs and control UC-MSCs were cultured in cell culture medium containing $0.4 \mu \mathrm{g} / \mathrm{mL}$ puromycin for 7 days to generate a stable cell lines. The screening effect was observed under the inverted fluorescence microscope. When the fluorescence positive rate was close to or higher than $99 \%$, the stable expression of CXCL9 gene of umbilical cord mesenchymal stem cell line was obtained.

\section{Western blot analysis of CXCL9 protein}

Western blot (WB) analysis was performed to examine levels of CXCL9 protein, in which the same number of CXCL9-UC-MSCs and control UC-MSCs (transfected with lenti-mCherry empty vector) were used to extract total protein samples. The concentration of protein was determined by BCA method (Thermo Fisher Scientific, MA, USA) following the manufacturer's instructions. Proteins were separated on sodium dodecyl sulfate-polyacrylamide gel electrophoresis (SDS-PAGE) and transferred to polyvinylidene fluoride (PVDF) membrane. The PVDF membrane was blocked in $5 \%$ skim milk and then incubated with the primary antibody (1:5000, Abcam) at $4^{\circ} \mathrm{C}$ overnights. After washing the PVDF membrane by TBST, it was incubated with the secondary antibody which is a horseradish peroxidase (HRP)-conjugated goat anti-rabbit IgG (1:3000, Abcam) for 50 minutes at room temperature. The resulting blots were detected by enhanced chemiluminescence $(E C L)$ and visualized under an imaging system(Thermo Fisher Scientific).

\section{Cell counting kit (CCK8) and flow cytometry assay of CXCL9-UC-MSCs and control UC-MSCs}


CXCL9-UC-MSCs and control UC-MSCs cells were cultured in 96 wells at a density of $1 \times 10^{4}$ cells per well respectively, and each group has 5 multiple wells. On the 1st-7th day after inoculation, 20 ul CCK-8 solution (Dojindo Molecular Technologies, Kumamoto, Japan) was added to each well and incubated at $37^{\circ} \mathrm{C}$ in a humidified atmosphere of $5 \% \mathrm{CO} 2$ for 4 hours. Then, the optical density (OD) value of each well was measured and recorded by an enzyme-labeled microplate spectrophotometer at $450 \mathrm{~nm}$. The Flow cytometry assay of CXCL9-UC-MSCs was performed as described above.

\section{Hepatogenic differentiation}

To further investigate whether overexpression of CXCL9 affects the differentiation of UC-MSCs into hepatocyte like cells, we induced CXCL9-UC-MSCs and control UC-MSCs into hepatocytes in vitro. The two kinds of cells were plated in the 6 well plates which had been coated with gelatin in advance, and the complete medium was added until about $70 \%-80 \%$ confluence. After that, the complete medium was replaced by hepatocyte induction medium. First, cells were cultured with IMDM (Sigma Chemical, MO, USA) containing EGF (20ng/ml, Novoprotien, Shanghai, China) and bFGF(10ng/ml, Novoprotien) for 2 days. Next, the IMDM containing HGF(20ng/ml, Novoprotien), bFGF(10ng/ml, Novoprotien) and nicotinamide $(0.61 \mathrm{~g} / \mathrm{L}$, Sigma Chemical, MO, USA) was used for 7 days' culture. During that time we exchanged the medium once. IMDM which was changed every $3-4$ days containing OSM $(20 \mathrm{ng} / \mathrm{ml}$, Novoprotien ), dexamethasone (1 umol/L, Beijing Solarbio Science \& Technology, Beijing,China) and ITS $(50 \mathrm{~g} / \mathrm{L}$, Sigma Chemical) was used for culture at last. At the fourth week of culture, the induced cells were stained with immunofluorescence respectively, and the fluorescent expression levels of hepatocyte specific proteins ALB(1:100, Abcam), AFP(1:100, Abcam) and CK18(1:200, Abcam) were observed under inverted fluorescence microscope (Tokoy, Japan, Nikon).

\section{Animal modle of liver fibrosis}

We purchased 55 male Sprague-Dawley rats aged 6 to 8 weeks from Beijing SiPeiFu Biotechnology Co., Ltd. All animals were raised and treated in specific pathogen-free (SPF)-grade cages in the Animal Center of the Fourth Hospital of Hebei Medical University. All animal experiments were approved by the Hebei Medical University Animal Care and Use Committee. Animal care and related procedures were approved by the Animal Care and Management Committee of the Fourth Hospital of Hebei Medical University. Liver fibrosis was induced by intraperitoneally (i.p.) injecting rats with CCL4 (3ml/kg; RHAWN, Shanghai, China) dissolved in oliver oil (Macklin, Shanghai, China), twice a week for 6 weeks (12 dose). We randomly selected 45 rats for modeling, and the remaining 10 were used as normal controls.

\section{Tansplantation of CXCL9-UC-MSCs and control UC-MSCs}

In the remaining 30 rats of liver fibosis group, ten rats were were injected CXCL9-UC-MSCs $\left(1 \times 10^{6}\right)$ via the tail vein after 3 days of the last dose of CCL4. In parallel with the treated group, twenty CCL4- induced rats were injected with control UC-MSCs $\left(1 \times 10^{6}\right)$ and PBS (200ul) instead of CXCL9-UC-MSCs as positive control group and negative control group. Moreover, another 10 rats were treated as normal controls without any treatment. The survival of rats in the four groups were observed and recorded daily. 


\section{Assessment of liver function}

The treatment effect was quantified by assessing serum levels of serum levels of alanine aminotransferase (ALT), aspartate aminotransferase (AST) and total bilirubin (T-Bil). In addition, hematoxylin-eosin (HE) and masson 's trichrome (MT) staining were used to observe the pathological changes of liver in each group. The serum of rats were determined in an automated biochemical analyser (Selectra 2, Merck, Darmstadt, Germany) 4 weeks after the initiation of the cell-based therapies. Animal liver tissues of each group were obtained after cardiac perfusion with ice saline. Next, tissues were fixed in $4 \%$ paraformaldehyde, embedded in paraffin, and sliced to a thickness of $4 \mu \mathrm{m}$. Slices were subsequently stained by HE and MT.

\section{Analysis of UC-MSCs homing ability to rat liver}

To compare the homing efficiency of CXCL9-UC-MSCs and control UC-MSCs to the injured rat liver,the frozen section technique were used. At the 24 hours and 4 weeks after cell therapies. We took out the rats' liver of each group, frozen the tissues in refrigerator at $-80^{\circ} \mathrm{C}$ immediately, embedded in optimal cutting temperature compound (OTC), frozen sections ( $8 \mu \mathrm{m}$ thick) were prepared to observe the accumulation and metabolism of mCherry in liver tissue under inverted fluorescence microscope ( Tokoy, Japan, Nikon).

\section{Statistical analysis}

Statistical analysis used a commercially available statistical software package IBM SPSS Statistics, version 23.0 for Windows (SPSS, Chicago, IL, USA). Statistical differences were analysed for significance by applying one-way ANOVA. $\mathrm{P}<0.05$ was considered statistically significant.

\section{Results}

\section{Characterization and differentiation ability of UC-MSCs}

About 7 days after umbilical cord tissue was isolated, some cells migrated from tissue fragment. Then, continued to culture and passage. UC-MSCs were confirmed successfully by their morphology under the inverted microscope (Fig.1a). Next, we characterized and validated the UC-MSCs at passage 3 by flow cytometry using specific cell surface markers for UC-MSCs. As shown in the figure, the positive expression rates of CD34 and CD 45 were both $0.5 \%, 98.2 \%$ of the cells were CD73-positive and $99.7 \%$ were CD90-positive (Fig.1b), indicating that the surface markers of UC-MSCs were consistent with expression characteristics. Meanwhile, after 3 weeks of adipogenic differentiation, the shape of the MSCs gradually became round or irregular, and bright red lipid droplets could be seen after Oil red 0 staining. Subesequently, UC-MSCs were induced for 4 weeks in the osteogenic differentiation medium, the morphology of cells were obviously changed from long fusiform to polygonal, and Alizarin red staining showed the calcium nodules (Fig.1C). This indicates that MSCs have multiple differentiation potentials.

\section{Preparation of CXCL9-UC-MSCs and Control UC-MSCs}


The screening results of pre-experiment confirm that $\mathrm{MOI}=50$ is the best transfection efficiency. Then, naive UC-MSCs at passage 4 were transfected with the lenti-CXCL9-mCherry and lenti-mCherry empty vector, and puromycin selection was completed after 7 days. Under the invert fluorescence microscopy, the UC-MSCs were tested positive for red fluorescence at 99\% approximately (Fig.2a), indicating successful genetic modification of the UC-MSCs. Western blot analysis has shown that CXCL9 protein was overexpressed markedly in CXCL9-UC-MSCs compared with control UC-MSCs (Fig.2b), demonstrating the successful establishment of the CXCL9-UC-MSCs cell line with stable CXCL9 overexpression.

\section{Growth trend and phenotype identification of CXCL9-UC-MSCs}

The result of CCK-8 analysis demonstrated that in the process of UC-MSCs cell proliferation, the 1st to the 3rd days is the incubation period of cell growth. Next, it entered logarithmic growth period at the 3rd to the 6th day. After that, due to the excessive number of cells, the cell growth entered the plateau phase, and the growth curve was S-shaped which was consistent with the regularity of cell growth. However, compared with the control UC-MSCs, the proliferation ability of the CXCL9-UC-MSCs was significantly decreased (Fig.2C). Afterwards, we identified CXCL9-UC-MSCs by flow cytometry, the results showed that the CD34-positive and CD45-positive rats were both $0.2 \%$, and the positive expression rates of CD73 and CD90 was $99.8 \%$ and $99.9 \%$, respectively (Fig.2d). This suggested that the overexpression of CXCL9 didn't affect the characterization of UC-MSCs, but it has a significant inhibitory effect on the proliferation of UC-MSCs.

\section{Comparison of the ability to differentiate into hepatocytes}

We used the same induction method to induce CXCL9-UC-MSCs and UC-MSCs into hepatocytes. About 12 days after induction, the morphology of the cells changed from long spindle to round or irregular polygon gradually, the nucleus was more obvious than before, which was similar to the morphological characteristics of hepatocytes, and the granules in the cytoplasm increased gradually (Fig.3). After 4 weeks of induction, we stained the cells with immunofluorescence. The results showed that the green fluorescence of CK18 was expressed in the cell membrane, AFP and ALB were expressed in the cytoplasm, and there was no significant difference between CXCL9-UC-MSCs and control UC-MSCs (Fig.4). Illustrating that the UC-MSCs overexpressing CXCL9 still had the ability to differentiate into hepatocyte like cells.

\section{Verification of CCl4-induced liver fibrosis}

We dissected the rats that received CCL4, we found that there is a severe adhesion between liver and other abdominal organs, the edge of the rat's liver was round and blunt, and the texture was harder than that of the normal liver. Haematoxylin-eosin (H\&E) staining and Masson's trichrome (MT) staining of the paraffin sections demonstrated that rat liver tissue has obvious necrosis, significant steatosis, and fibrous structure disorder, fibrous tissue intersperses to separate liver lobules, and pseudo-lobules have 
formed. These observations are in accordance with the histological characteristics of liver fibrosis, and the fibrosis scored as grade 4 based on Knodell's scoring system (Table.1).

\section{Recovery of the liver in the CCl4-induced fibrosis rat model after transplantation CXCL9-UC-MSCs}

H\&E staining and MT staining showed the morphological changes and level of hepatic damage in the livers of rats with liver fibrosis after transplantation. Histologically the degeneration and necrosis of hepatocyte in the CXCL9-UC-MSCs and UC-MSCs livers were less severe than in the PBS-treated pathologic control group. However, there was less degeneration and necrosis in the CXCL9-UC-MSCs group. Moreover, in the CXCL9-UC-MSCs group, we observed that the content of collagen fibers around and inside the hepatic lobules decreased obviously, the pseudo-lobules almost disappeared, and the morphology of hepatic lobules was basically normal, the ballooning degeneration of hepatocytes was also greatly improved (Fig.5a). Both H\&E and MT staining indicated that the infusion of CXCL9-UC-MSCs into rats with CCL4-induced fibrosis improved the histological structure of the liver compared with the control treatment. Meanwhile, the activities of serum alanine aminotransferase (ALT), aspartate aminotransferase (AST) and total bilirubin (T-Bil) were measured with automatic biochemistry analyser. The ALT, AST and T-Bil has highly increased in the PBS group relative to the normal group. However, the levels were significantly relieve in the CXCL9-UC-MSCs and UC-MSCs groups 4 weeks after initiation of cell transplantation (Fig.5b).

\section{Enhanced ability of homing in CXCL9-UC-MSCs to the liver of rats with liver fibrosis}

The liver of rats in each group were collected after 24 hours and 4 weeks of cell transplantation for frozen sections. Under the fluorescent inverted microscope, 24 hours after the injection of cells, the mCherrylabeled cells in the livers of CXCL9-UC-MSCs and control UC-MSCs groups were scattered in the liver lobules, mainly concentrated around the blood vessels, and the fluorescence of CXCL9-UC-MSCs group was significantly more than that of control UC-MSCs group. Four weeks later, the CXCL9-UC-MSCs group still had more mCherry fluorescence expression, but the control UC-MSCs fluorescence expression was very weak (Fig.6). These results indicate that CXCL9-UC-MSCs have stronger chemotaxis than control UCMSCs, and can stay longer in the fibrotic liver tissue and play a stronger anti-fibrotic role.

\section{Discussion}

The effectiveness of CXCL9-expressing UC-MSCs in suppressing liver fibrosis and stimulating liver regeneration in vitro and in vivo shows that CXCL9-UC-MSCs have a powerful therapeutic effect in rats with CCl4-induced liver fibrosis. The major findings are as follows:(1) The expression of CXCL9 did not affect the characterization of UC-MSCs, nor does it affect the ability of UC-MSCs to differentiate into hepatocyte like cells, but had an effect on the growth and proliferation of UC-MSCs; (2) transplantation of CXCL9-UC-MSCs greatly ameliorated liver injury, and the effects of CXCL9-UC-MSCs exceeded those of control UC-MSCs; (3) treatment with CXCL9-UC-MSCs reversed liver damage and decreased toxicity, seen as reduction of ALT, AST and T-Bil back to normal levels; and (4) CXCL9-UC-MSCs homed into fibrosisaffected rat liver more efficiently than control UC-MSCs. 
Transplantation of liver progenitor cells (LPCs), mesenchymal stem cells (MSCs) and hematopoietic stem cells (HSCs) has become a promising therapy to treat various liver disease mainly due to the ability of these cell types to promote liver regeneration $[9,10]$. Among the that, MSCs have the characteristics of multi-directional differentiation potential, low immunogenicity, and non-induction of tumorigenesis even after multiple passages in vitro [11-13]. Human MSCs, can be derived from bone marrow (BM-MSCs), adipose tissue (AT-MSCs), amniotic fluid (AF-MSCs), dental pulp (DP-MSCs), umbilical cord (UC-MSCs) and fetal lung (FL-MSCs) [14]. UC-MSCs and BM-MSCs are widely used in liver injured diseases, but UCMSCs have the advantages of convenient source, lower immunogenicity and less ethical restrictions compare to BM-MSCs. Therefore, UC-MSCs are the cell source in this study.

Most chronic liver diseases involving inflammation have a fibrotic component which involves remodeling and excess accumulation of extracellular matrix components (EMC), and fibrosis leads to liver failure and death eventually. In this study, the liver fibrosis rat model was generated by intraperitoneal injection of CCL4 as previously reported [15]. In rencent years, MSCs transplantation has been considered as a promising approach for the treatment of liver fibrosis [16-18]. In vitro and in vivo experiments demonstrated that MSCs mainly exert antifibrotic effects by paracrine mechanisms [16]. MSCs can produce a range of cytokines and growth factors, such as insulin-like growth factor-1 (IGF-1), epidermal growth factor (EGF), hepatocyte growth factor (HGF), and milk fat globule-EGF factor-8 (MFGE8) which have been shown to promote hepatocyte regeneration in situ, inhibit apoptosis of hepatocyte, suppress inflammatory responses, affect HSC activation, and promote the degradation of extracellular matrix [1922]. Moreover, MSCs can differentiate into hepatocyte-like cells and are suitable for transfection with exogenous genes [23]. However, the treatment effect of MSCs transplantation is not very satisfactory, the main reason is lacking of enough cells to function in the injured sites of liver after injected into the body.

The CXCL9 is a famous member of the CXC chemokine family and a potent chemoattractant for activated T lymphocytes, natural killer cells and monocytes, participating recruitment of Th1 regulatory $T$ cells[24]. In addition to immune responses of Th1, CXCL9 and its receptor, CXCR3 has also been reported to be involved in the occurrence of chronic diseases in lung and liver [7,25,26-29]. As we known, the occurrence of liver fibrosis is closely related to the activation and proliferation of HSC and the upregulation of the expression of vascular endothelial growth factor (VEGF). Activated HSCs are the major source of collagen in the liver, which can secrete a large amount of ECM protein, tissue inhibitors of metalloproteinases and matrix metalloproteinases (MMPs), causing the remodeling of liver structure subsequently[30,31]. Interestingly, increasing concentrations of CXCL9 lead to dose-dependent downregulation of transforming growth factor $\beta 1$ (TGF- $\beta 1$ )and collagen 1a1mRNA, which are playing an important role in the process of liver forosis. Furthermore, collagen protein expression of stellate cells can be strongly suppressed by CXCL9 [7]. VEGF is a central angiogenic factor during chronic liver injury. In the process of fibrosis formation, VEGF promotes the formation of new blood vessels and accelerates the remodeling process of liver lobules[32]. However, CXCL9 can be used as an anti-fibrotic cytokine together with metalloproteinase 13 (MMP13) to achieve an anti-fibrotic effect by regulating the expression of VEGF[33]. Recently, some studies have shown that the CXCL9/CXCR3 signaling pathway can promote cell migration, and proposed that MSCs could interact with aortic endothelial cells showing firm adhesion, 
and crawling and spreading behaviour, which were all enhanced by CXCL9 $[34,35]$. But some researchers suggested that the antifibrotic action of CXCL9 might not be strong enough to ultimately cause a reduction in fibrosis in human diseases[7]. Therefore, we hypothesized that overexpression of CXCL9 could improve the therapeutic efficacy of UC-MSCs transplantation for the treatment of liver fibrosis, and consequently promote the homing capabilities of UC-MSCs to the rat livers affected by fibrosis.

The purpose of this study is to combine UC-MSCs and chemokines with genetic engineering technology, so that they can play a stronger role in anti-fibrosis. Extensive studies have suggested that gene therapy is likely to become an option for the treatment of many forms of liver diseases, and may ameliorate patient care, survival, and prognosis[36,37]. Lentiviral vector is one of the most widely used gene delivery system, the vector can effectively deliver foreign genes into host chromosomes, so as to achieve stable expression. In terms of infection ability, it can highly efficient infect neurons, hepatocytes, cardiomyocytes, tumor cells, endothelial cells, stem cells and other types of cells, so as to achieve good gene therapy effect[38]. Considering the advantages of the lentivirus as a highly efficient gene transfection system for gene therapy, the lentivirus vector was selected in this study to deliver the CXCL9 gene into UC-MSCs. Our results validated that after puromycin screening, the transfected UC-MSCs could persistently expressing CXCL9.

In this study, the cell therapy and gene therapy were combined to achieve a better anti-fibrosis effect. And this combination will not affect the characteristics of UC-MSCs, such as the ability to differentiate into hepatocytes. As expected, when the CXCL9-UC-MSCs and control UC-MSCs transplantation groups were compared, we found that liver fibrosis rats in the CXCL9-UC-MSCs transplantation group experienced better therapeutic effects as assessed by markers for liver function and liver pathology. The liver function of the CXCL9-UC-MSCs group was significantly improved, and the fibrosis was also obviously different than that of the control UC-MSCs group. Besides, we detected the levels of liver enzymes such as ALT, AST and T-Bil in serum 4 weeks after the initiation of cell therapy, and found that their levels were notablely decreased in both the CXCL9-UC-MSCs and control UC-MSCs groups. The AST, AST and T-Bil levels decreased to normal levels in the CXCL9-UC-MSCs-treated group, whereas they were slightly higher than normal in the control UC-MSCs-treated group. Moreover, from the results of frozen sections, the homing ability of CXCL9-UC-MSCs and the residence time in the liver are indeed stronger and longer than those of the control UC-MSCs group. These results clearly indicated that the transplantation of UC-MSCs have a therapeutic effect, and the transplantation of CXCL9-UC-MSCs has a synergistic regenerative effect on fibrotic livers. Regeneration may be related to the promotion of UC-MSCs migration and incorporation into liver tissue.

This study still has several limitations. First, CXCL9 has undermined the growth and proliferation of UCMSCs, further studies are underway in our laboratory to characterize CXCL9-UC-MSCs-secreted cytokines in cell culture. Second, we have only observed that the CXCL9-expressing UC-MSCs can improve liver fibrosis better, but we have not discussed the exact mechanism yet. At last, the microenvironment of liver fibrosis cannot be fully replicated in vitro, so whether CXCL9 affects the differentiation of UC-MSCs into hepatocyte like cells in vivo needs to be further explored. 


\section{Conclusion}

In conclusion, our results demonstrated that overexpression of CXCL9 improves the efficacy of UC-MSCs therapy for the repair of liver fibrosis, thereby promoting homing of UC-MSCs to the injured liver of rats with liver fibrosis.

\section{Abbreviations}

UC-MSCs: Umbilical cord mesenchymal stem cells; MSCs: mesenchymal stem cells; OLT: Orthotopic liver transplantation; HSC: hepatic stellate cells; VEGF: vascular endothelial growth factor; PBS: phosphatebuffered saline; SDS-PAGE: sodium dodecyl sulfate-polyacrylamide gel electrophoresis; PVDF: polyvinylidene fluoride; HRP: horseradish peroxidase; ECL: enhanced chemiluminescence; CCK8: Cell counting kit; OD: optical density; bFGF: basic fibroblast growth factor; IMDM: Iscove's Modified Dulbecco's Medium; HGF: hepatocyte growth factor; OSM: oncostatin M; ALB: albumin; AFP: alpha fetoprotein; CK18: Cytokeratin 18; SPF: specific pathogen-free; i.p.: intraperitoneally; ALT: alanine aminotransferase; AST: aspartate aminotransferase; T-Bil: total bilirubin; HE: hematoxylin-eosin; MT: masson 's trichrome; OTC: optimal cutting temperature compound; SPSS: Statistical Package for the Social Sciences; LPCs: liver progenitor cells; HSCs: hematopoietic stem cells; BM-MSCs: bone marrow mesenchymal stem cells; ATMSCs: adipose tissue mesenchymal stem cells; DP-MSCs: dental pulp mesenchymal stem cells; FLMSCs: fetal lung mesenchymal stem cells; EMC: extracellular matrix components; IGF-1: insulin-like growth factor-1; EGF: epidermal growth factor; HGF: hepatocyte growth factor; MFGE8: milk fat globuleEGF factor-8; MMPs: matrix metalloproteinases.

\section{Declarations}

\section{Ethics approval and consent to participate}

Human umbilical cord were obtained from Hebei General Hospital with patients' informed consent, and in compliance with national legislation. All experimental protocols were approved by the Ethics Committee of Hebei General Hospital. (protocol number: 2019224).

All animal experiments were approved by the Animal Care and Use Committee of the Fourth Hospital of Hebei Medical University. (protocol number:W2021001).

\section{Consent for publication}

Not applicable

\section{Availability of data and materials}

All data generated or analyzed supporting conclusions are included in the current manuscript. 
The authors declare that they have no competing interests.

\section{Funding}

This work was supported by the Natural Science Foundation of Hebei province, China. (No.21372406D).

\section{Author contributions}

Yang Li: Design of the study, Data curation, Formal analysis, Writing original draft. Xueshuai Ye: Contributed to the culture of UC-MSCs, Western blot, and flow cytometry. Xueqian Zhang: Data curation, Animal experiments. Ziqi Cai: Data curation, Writing-review \& editing. Li Shen and Wentao Zhang: Assisted with the isolation, culture, and characterization of stem cells. Mengya Zhang: Participated in the Western blot experiments. Jianhui Cai: Funding acquisition, Supervision. All authors approved the final version of the manuscript to be published.

\section{Acknowledgments}

The authors would like to thank Dr. Zhengqin Sun and Dr. Liying Chen for their technical guidance. They also thank Yilin Deng for editing the manuscript.

\section{References}

1. Parekkadan B, van Poll D, Suganuma K, etal. Mesenchymal stem cell-derived molecules reverse fulminant hepatic failure. PloS ONE. 2007; e941: 1-6.

2. Ishikawa T, Terai $\mathrm{S}$, Urata $\mathrm{Y}$, et al. Administration of fibroblast growth factor 2 in combination with bone marrow transplantation synergistically improves carbon-tetrachloride-induced liver fibrosis in mice. Cell Tissue Res. 2007; 327:463-470.

3. Kharaziha P, Hellstrom PM, Noorinayer B,et al. Improvement of liver function in liver cirrhosis patients after autologous mesenchymal stem cell injection: a phasel-II clinical trial. Eur J Gastroenterol Hepatol. 2009; 21:1199-1205.

4. Xagorari A, Siotou E, Yiangou M, et al.Protective effect of mesenchymal stem cell-conditioned medium on hepatic cell apoptosis after acute liver injury. Int J Clin Exp Pathol. 2013; 6:831-840.

5. Lee KD, Kuo TKC, Whang-Peng J, et al. In vitro hepatic differentiation of human mesenchymal stem cells. Hepatology. 2004; 40: 1275-1284.

6. Ya Yang, Yalei Zhao, Lingjian Zhang, Fen Zhang, Lanjuan Li. The Application of Mesenchymal Stem Cells in the Treatment of Liver Diseases: Mechanism, Efficacy, and Safety Issues. Front Med (Lausanne).2021; May 31;8:655268.

7. Wasmuth HE, Lammert F, Zaldivar MM, et al. Antifibrotic effects of CXCL9 and its receptor CXCR3 in livers of mice and humans. Gastroenterology. 2009;137:309-319:319e1-e3.

8. Chamberlain G, Smith H, Rainger GE, Middleton J. Mesenchymal Stem Cells Exhibit Firm Adhesion, Crawling, Spreading and Transmigration across Aortic Endothelial Cells: Effects of 
Chemokines and Shear. PLoS One.2011;6(9):e25663.

9. Tsolalo E, Yannaki E. Stem cell-based regenerative opportunities for the liver:state of the art and beyond. World J Gastroenterol. 2015;21(43):12334-50.

10. Lorenzini S, Gitto S, Grandini E, Andreone P, Bernardi M. Stem cells for end stage liver disease: how far have we got? World J Gastroenterol. 2008;14(29): 4593-99.

11. Bongso A, Fong CY. The therapeutic potential, challenges and future clinical directions of stem cells from the Wharton's jelly of the human umbilical cord [J]. Stem Cell Rev. 2013; 9(2): 226-240.

12. Fan CG, Zhang QJ, Zhou JR. Therapeutic potentials of mesenchymal stem cells derived from human umbilical cord [J]. Stem Cell Rev. 2011; 7(1):195-207.

13. Vidal MA, Walker NJ, Napoli E, et al. Evaluation of senescence in mesenchymal stem cells isolated from equine bone marrow, adipose tissue, and umbilical cord tissue [J]. Stem Cells Dev. 2012; 21 (2): 273-283.

14. Volarevic V, Nurkovic J, Arsenijevic N, Stojkovic M. Concise Review: Therapeutic Potential of Mesenchymal Stem Cells for the Treatment of Acute Liver Failure and Cirrhosis. Stem Cells. 2014; Nov ;32(11):2818-23.

15. Ghallab, A; Myllys, M; Holland, CH; Hengstler, JG, et al. Influence of Liver Fibrosis on Lobular Zonation. Cells. 2019 Dec 2;8(12):1556.

16. Berardis S, Dwisthi Sattwika P, Najimi M, Sokal EM. Use of mesenchymal stem cells to treat liver fibrosis: current situation and future prospects. World J Gastroenterol. 2015; 21:742-58.

17. Hu C, Zhao L, Zhang L, Bao Q, Li L. Mesenchymal stem cell-based cell-free strategies: safe and effective treatments for liver injury. Stem Cell Res Ther. 2020; 11:377.

18. Xuan J, Feng W, An ZT, Yang J, Xu HB, Li J, et al. Anti-TGFß-1 receptor inhibitor mediates the efficacy of the human umbilical cord mesenchymal stem cells against liver fibrosis through TGFbeta-1/Smad pathway. Mol Cell Biochem. 2017; 429:113-22.

19. Kun Wang, Yuwen Li, Tiantian Zhu, Yongting Zhang, Wenting Li, Wenyu Lin, Jun Li, Chuanlong Zhu .Overexpression of c-Met in bone marrow mesenchymal stem cells improves their effectiveness in homing and repair of acute liver failure. Stem Cell Res Ther. 2017; Jul 5;8(1):162.

20. de Witte SFH, Merino AM, Franquesa M, Hoogduijn MJ, et al. Cytokine treatment optimises the immunotherapeutic effects of umbilical cord-derived MSC for treatment of inflammatory liver disease. Stem Cell Res Ther. 2017; Jun 8;8(1):140.

21. Berardis S, Dwisthi Sattwika P, Najimi M, Sokal EM. Use of mesenchymal stem cells to treat liver fibrosis: current situation and future prospects. World J Gastroenterol. 2015; 21:742-58.

22. An SY, Jang YJ, Lim HJ, Han J, Lee J, Lee G, et al. Milk fat globule-EGF factor 8, secreted by mesenchymal stem cells, protects against liver fibrosis in mice Gastroenterology. 2017; 152:117486.

23. Hong SH, Gang EJ, Jeong JA, Ahn C, Hwang SH, Yang IH, Park HK, Han H, Kim H. In vitro differentiation of human umbilical cord blood-derived mesenchymal stem cells into hepatocyte-like 
cells. Biochem Biophys Res Commun. 2005;330:1153-61.

24. Strieter RM, Burdick MD, Gomperts BN, et al. CXC chemokines in angiogenesis. Cytokine Growth Factor Rev. 2005;16:593-609.

25. Sahin H, Borkham-Kamphorst E, Kuppe C, et al. Chemokine Cxcl9 attenuates liver fibrosis-associated angiogenesis in mice. Hepatology 2012;55:1610-1619.

26. Medoff BD, Wain JC, Seung E, et al. CXCR3 and its ligands in a murine model of obliterative bronchiolitis: regulation and function. J Immunol. 2006;176:7087-95.

27. Zeremski M, Dimova R, Brown Q, Jacobson IM, Markatou M, Talal AH. Peripheral CXCR3-associated chemokines as biomarkers of fibrosis in chronic hepatitis $C$ virus infection. $J$ Infect Dis. 2009;200:1774-80.

28. Chuang YH, Lian ZX, Cheng CM, et al. Increased levels of chemokine receptor CXCR3 and chemokines IP-10 and MIG in patients with primary biliary cirrhosis and their first degree relatives. J Autoimmun. 2005;25:126-32.

29. Tacke F, Zimmermann HW, Berres ML, et al. Serum chemokine receptor CXCR3 ligands are associated with progression, organ dysfunction and complications of chronic liver diseases. Liver Int. 2011;31:840-9.

30. Li D, He L, Guo H, Chen H, Shan H. Targeting activated hepatic stellate cells (aHSCs) for liver fibrosis imaging. EJNMMI Res 2015;5:71.

31. Puche JE, Saiman Y, Friedman SL. Hepatic stellate cells and liver fibrosis. Compr Physiol .2013; 3: 1473-1492.

32. Tsuchida T, Friedman SL. Mechanisms of hepatic stellate cell activation. Nat Rev Gastroenterol Hepatol.2017 Jul ;14(7) :397-411.

33. Yang L, Kwon J, Popov Y, Shah V, et al. Vascular Endothelial Growth Factor Promotes Fibrosis Resolution and Repair in Mice. Gastroenterology. 2014 May;146(5) :1339-50.e1.

34. Zhu MX, Wan WL, Hong Y, Jing HM, et al. Expression and role of MIG/CXCR3 axis in mantle cell lymphoma. Exp Cell Res. $2020 ; 1215 ; 397(2)$.

35. Chamberlain G, Smith H, Rainger GE, Middleton J. Mesenchymal stem cells exhibit firm adhesion, crawling, spreading and transmigration across aortic endothelial cells effects of chemokines and shear. PLoS One. $2011 ; 6(9)$ :e25663.

36. Booth C, Gaspar HB, Thrasher AJ. Treating Immunodeficiency through HSC gene therapy. Trends Mol Med. 2016;22(4):317-27.

37. Moscoso CG, Steer CJ. The Evolution of Gene Therapy in the Treatment of Metabolic Liver Diseases. Genes (Basel). 2020; $0810 ; 11(8)$.

38. Rubinson DA, Dillon CP, Kwiatkowski AV, Sievers C, Yang L, Kopinja J, Rooney DL, Zhang M, Ihriq MM, McManus MT, Gertler FB, Scott ML, Van Parijs L. A lentivirus based system to functionally silence genes in primary mammalian cells, stem cells and transgenic mice by RNA interference. Nat Genet. 2013;33(3):401-6. 


\section{Tables}

Table1 Degree of fibrosis based on Knodell's scoring system

\begin{tabular}{ll}
\hline Stage score & Description \\
\hline 1 & No fibrosis \\
2 & Fibrous pericentral expansion \\
3 & Bridging fibrosis \\
4 & Fibrosis septa and structural disturbance of hepatic lobule \\
5 & Cirrhosis \\
\hline
\end{tabular}

Figures

a

P0
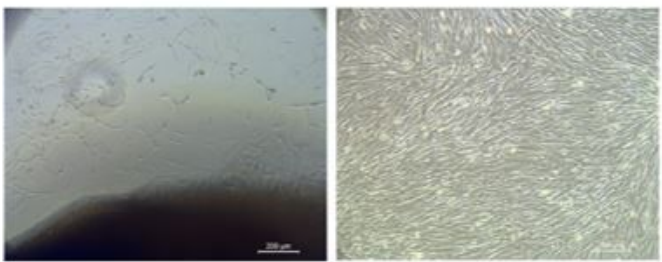

Osteogenic induction

C Adipogenic induction
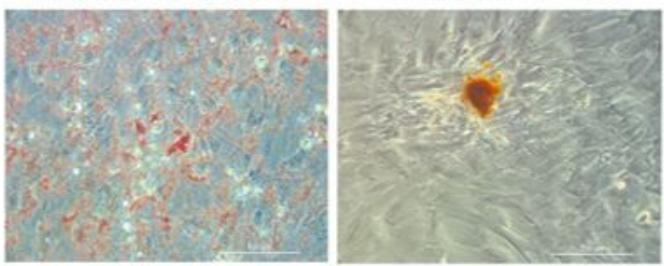

b

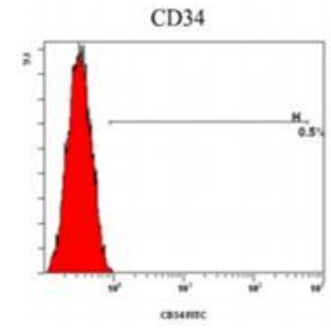

CD90

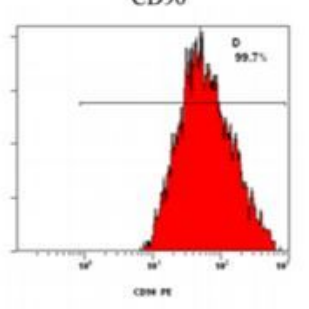

CD45

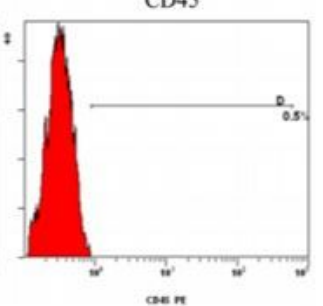

Control-FITC

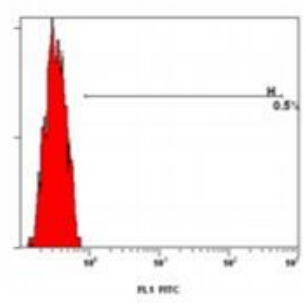

$\mathrm{CD} 73$

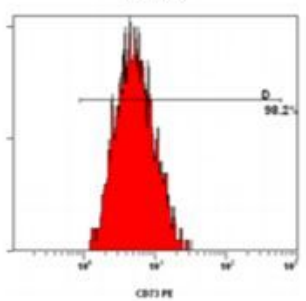

Control-PE

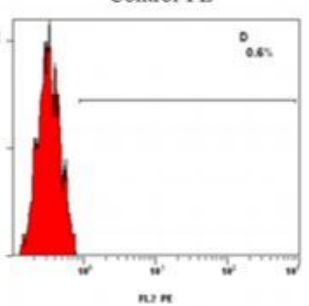

\section{Figure 1}

UC-MSCs culture detection and differentiation ability. a The primary umbilical cord mesenchymal stem cells (UC-MSCs) crawled out from the edge of the tissue block, and the morphology of the cells was uniform spindle or fibroblast in the third generation $(\times 100)$. b Determination of the naive UC-MSCs phenotypes by flow cytometry. c Functional characterization of UC-MSCs. In adipocyte-differentiated cells, the accumulation of oil red 0-stained lipid drops was observed $(\times 400)$. UC-MSCs differentiated into osteocytes and exhibited enhanced calcium deposition by alizarin red $S$ staining $(\times 400)$. 
a
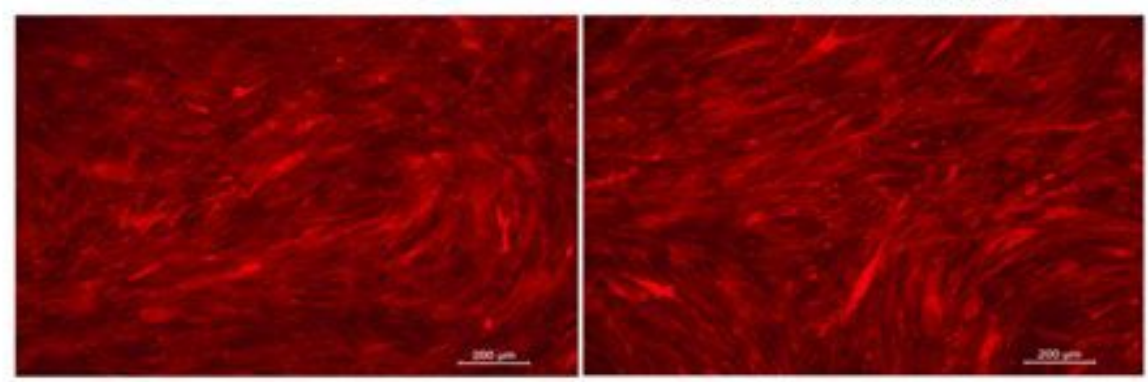

b

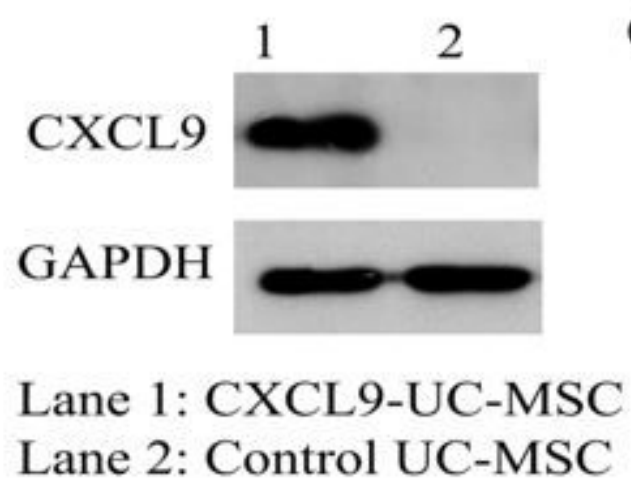

C

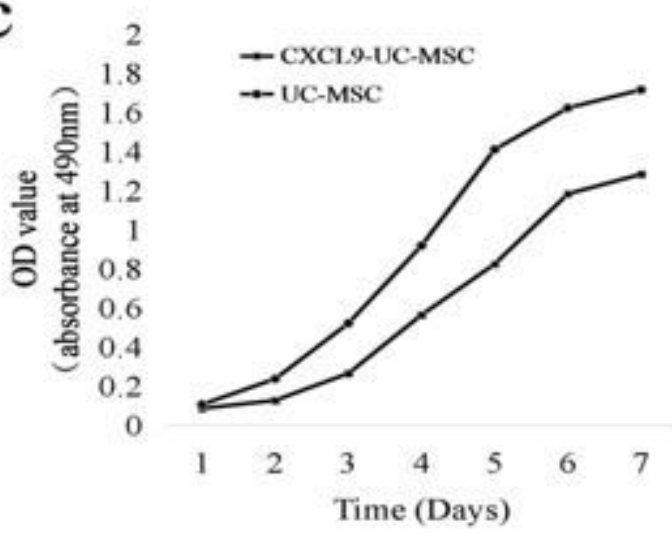

d
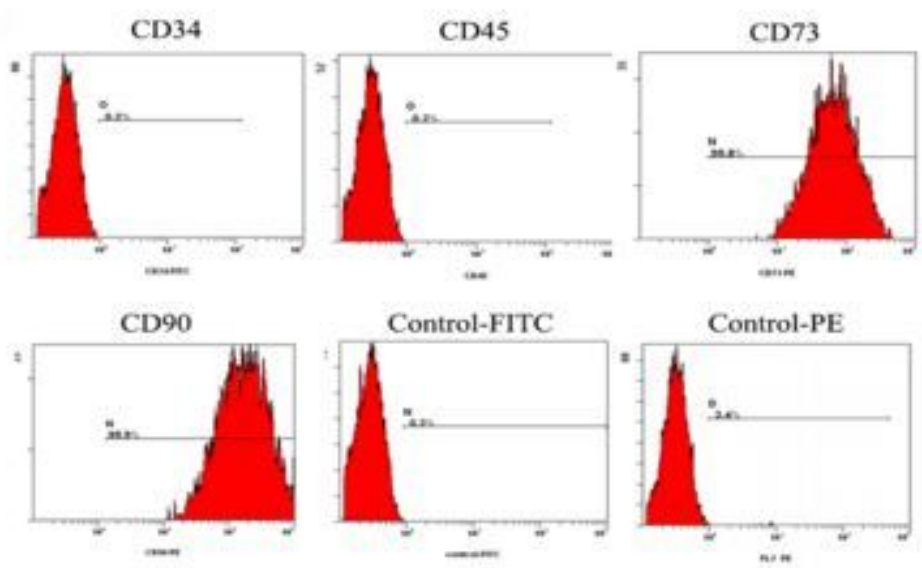

\section{Figure 2}

Overexpression of CXCL9 in UC-MSCs transfected with lenti-CXCL9-mCherry vector. The UC-MSCs were stably transfected with lenti-CXCL9-mCherry or lenti-mCherry empty vector, and then analyzed for characteristics. a Transfection efficiency was detected by invert fluorescence microscopy $(\times 100)$ in CXCL9-UC-MSCs and control UC-MSCs. b Western blot analysis of CXCL9 protein expression in CXCL9UC-MSCs and control UC-MSCs. c Comparing the growth and development of CXCL9-UC-MSCs and 
control UC-MSCs, the growth rate of CXCL9-UC-MSCs is significantly limited. $d$ Determination of the CXCL9-UC-MSCs phenotypes by flow cytometry.

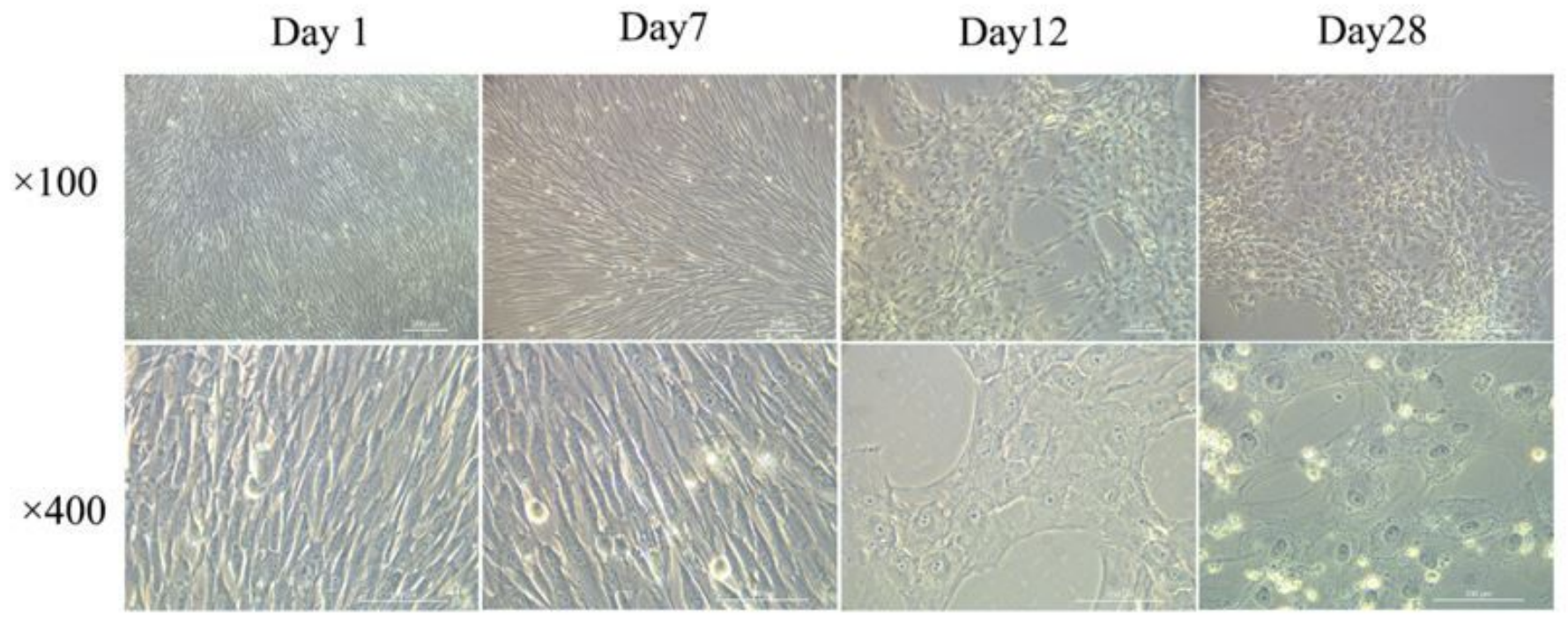

\section{Figure 3}

Changes of cell morphology during hepatogenic induction of UC-MSCs. With the prolongation of induction time, the shape of cells gradually changed from long spindle to irregular shape, the nucleus gradually became larger and more obvious, and the granules in cytoplasm increased significantly. 


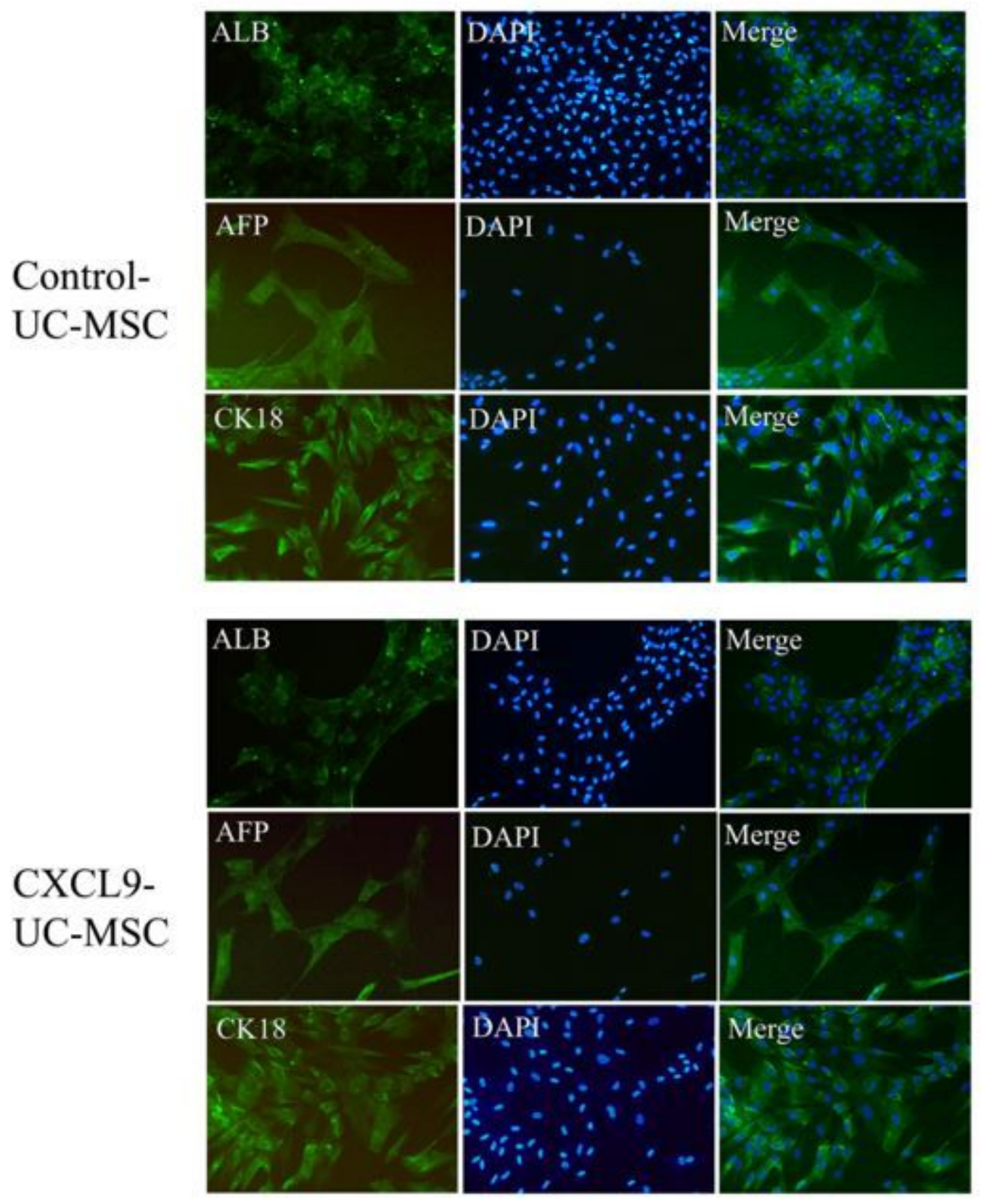

Figure 4

Immunofluorescence staining after hepatogenesis induction. Using the same induction method to induce CXCL9-UC-MSCs and control UC-MSCs. Immunofluorescence staining performed 28 days after induction demonstranted that CXCL9-UC-MSCs and control UC-MSCs all expressed hepatocyte-specific protein ALB, AFP and CK18 (×200). 

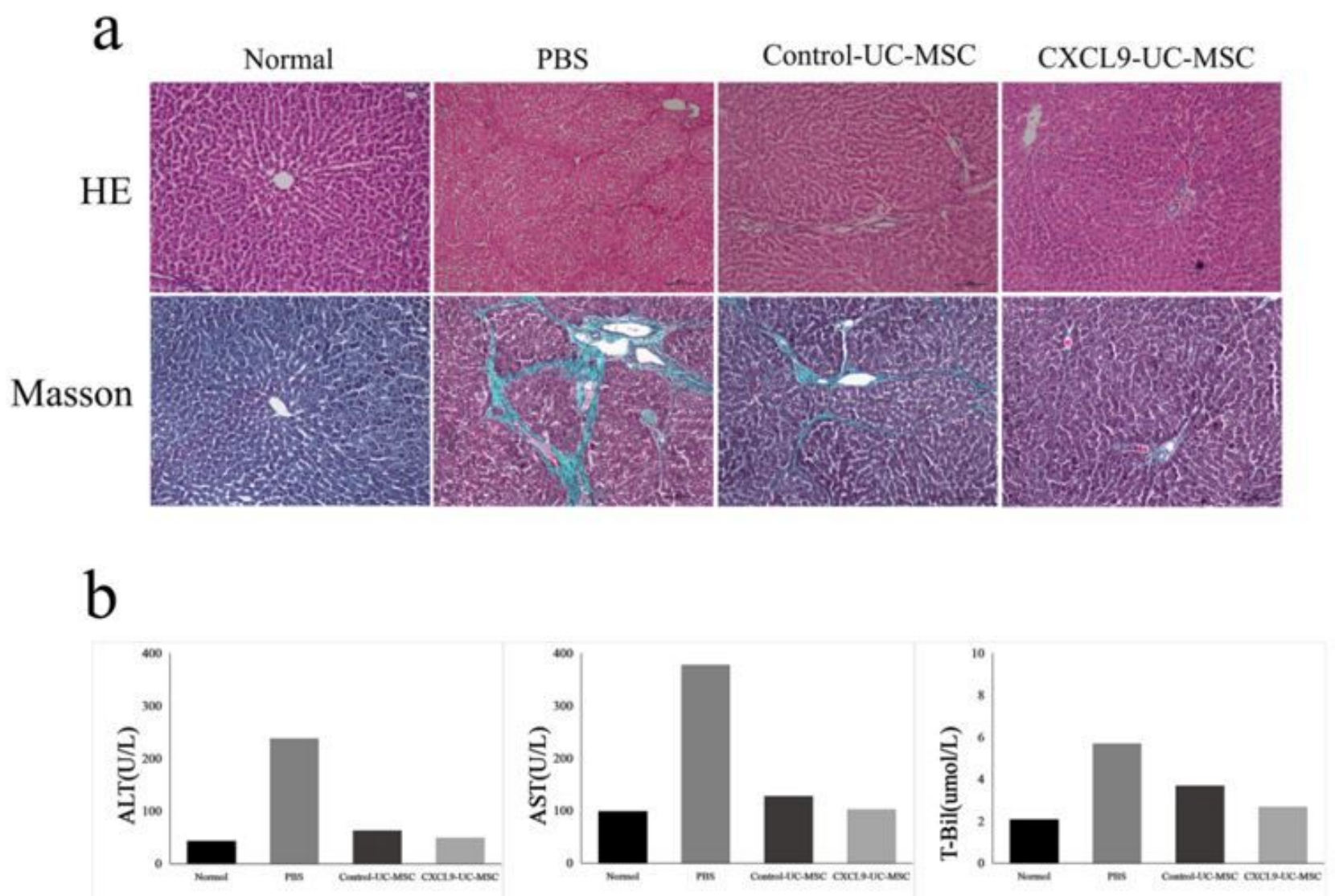

Figure 5

Effects of CXCL9-UC-MSC transplantation on liver pathology and liver function of rats with liver fibrosis. A total of $30 \mathrm{CCL} 4$-induced $(3 \mathrm{ml} / \mathrm{kg})$ rats were randomly divided into three groups: CXCL9-UC-MSCs, control UC-MSCs and PBS groups ( $n=10$ in each group). After 3 days of the last dose of CCL4, the CXCL9UC-MSCs and control UC-MSCs groups were given a transfusion of $1.0 \times 106$ cells suspended in 500 ul of PBS respectively, while the PBS group was given 500ul of PBS. Moreover, another 10 rats were treated as normal controls without any treatment. a 4 weeks later, the liver tissues of each groups were collected and subseqeuntly HE and MT stained $(\times 100)$. b The rates in the CXCL9-UC-MSCs, control UC-MSCs, PBS and normol groups, levels of serum alanine transferase (ALT), aspartate aminotransferase (AST), and total bilirubin (T-Bil) in treatment of liver fibrosis rats. Data were expressed as mean \pm SD. P『0.05. 


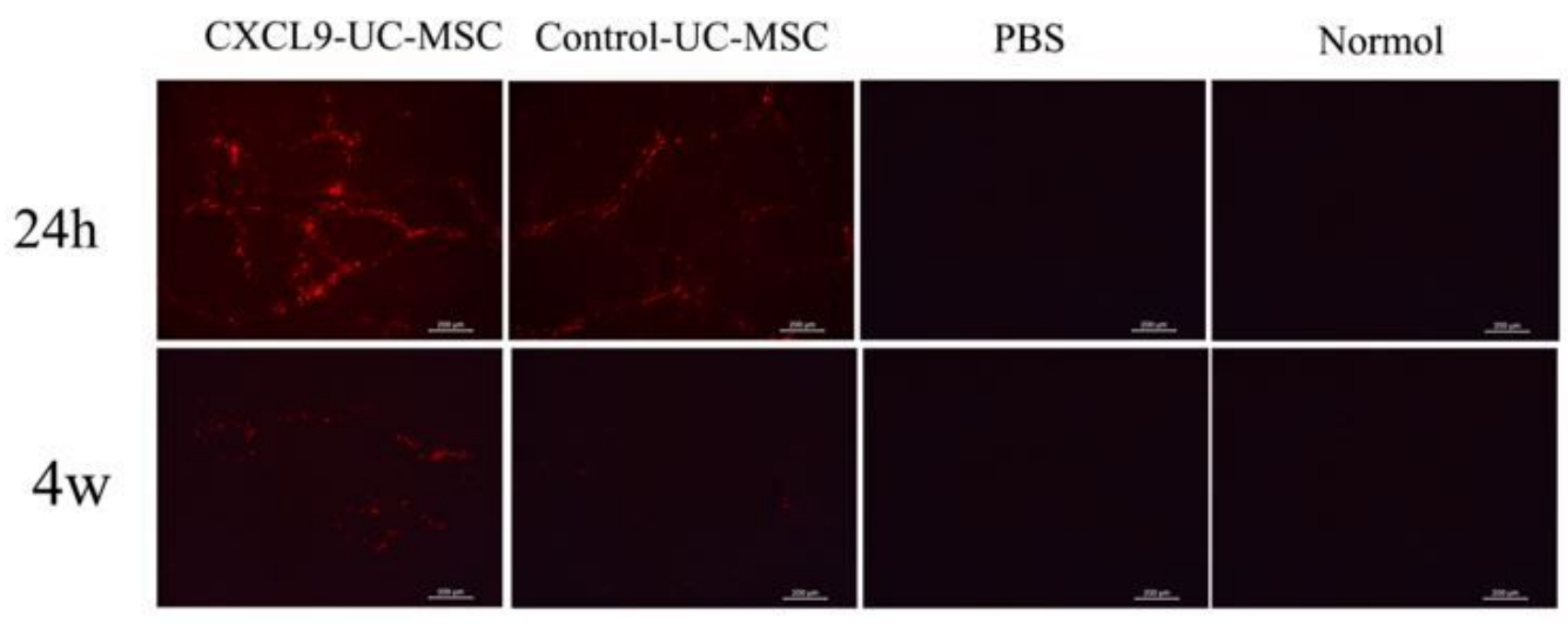

\section{Figure 6}

Analysis of cell migration in liver fibrosis rats transplanted with CXCL9-UC-MSCs using the frozen sections. 24 hours after the cell injection, 4 rats were randomly selected from each of the control UCMSCs, cell CXCL9-UC-MSCs, PBS and normol groups. The rats were sacrificed and the liver was prepared as frozen sections, the fluorescence expression was observed under inverted fluorescence microscope $(\times 100)$. After 4 weeks of cell therapy, the remaining rats in each group were dissected for HE and MT staining of liver. At the meanwhile, the frozen sections were performed again to observe the fluorescence expression $(\times 100)$. 